\title{
RPA provides checkpoint-independent cell cycle arrest and prevents recombination at uncapped telomeres of Saccharomyces cerevisiae
}

Nathalie Grandin ${ }^{1,2}$ and Michel Charbonneau ${ }^{1,2, *}$

1 UMR CNRS 5239; Ecole Normale Supérieure de Lyon; IFR128 BioSciences Gerland; 69364 Lyon; France; ${ }^{2}$ UMR CNRS 7292; Université François Rabelais de Tours; 37200 Tours, France

Running title: RPA and telomere end protection

Key words: budding yeast/cdc13-1 mutation/checkpoint-mediated cell cycle control/Replication Protein A/telomeric DNA damage

* Corresponding author:

Michel CHARBONNEAU ; UMR CNRS 7292 ; UFR Pharmacy ; University of Tours ; Parc Grandmont ; 31 avenue Monge ; 37200 TOURS, FRANCE

e-mail: Michel.Charbonneau@univ-tours.fr

Tel.: (33) 247366084

FAX: (33) 247367042 


\section{ABSTRACT}

Replication Protein A (RPA) is an evolutionary conserved essential complex with singlestranded DNA binding properties that has been implicated in numerous DNA transactions. At damaged telomeres, Saccharomyces cerevisiae RPA recruits the Mec1-Ddc2 module of the DNA damage checkpoint network, its only known function in DNA damage signaling. Here, we describe rfa1 mutants (rfa1-1, rfa1-9, rfa1-10, rfa1-11 and rfa1-12) that are proficient in this checkpoint but nevertheless exhibit deregulation of cell cycle control upon telomere uncapping induced by the cdc13-1 mutation. Overriding of this damage-induced checkpointindependent cell cycle block in the rfa1 mutants was suppressed following genetic inactivation of either TEL1 or EST2/telomerase. Altogether, our results suggest that a previously non suspected function of RPA is to block cell cycle progression upon telomere uncapping using a yet unidentified pathway that functions in a Mec1-Ddc2-independent manner. We propose that in the rfa1 mutants, ill-masking of uncapped telomeres provokes inappropriate access of Tel1 and inappropriate functioning of telomerase, which, by yet unknown mechanisms, allows cell division to take place in spite of the block established by the DNA damage checkpoint. In the present study, we also observed that upon telomere uncapping, rfa1-12, but not the other studied rfa1 mutants, triggered telomeric recombination in the presence of functional telomerase. In conclusion, the present study identifies a novel pathway of telomere end protection that utilizes a previously unsuspected function of RPA at the telomeres. 


\section{Introduction}

Early in their discovery, telomeres, the extremities of eukaryotic linear chromosomes, were regarded as a protective cap that prevented fusion between chromosomes [1,2]. Confirming these early assumptions, it is now still widely accepted that the main function of telomeres is to protect the genome against the occurrence of all events that normally promote repair of intrachromosomal DNA breaks, homologous recombination and non homologous end-joining (NHEJ), principally, including DNA damage signaling and checkpoint operation [3,4]. It is worth stressing that the second main function of telomeres, namely recruiting telomerase for telomere replication, also represents a sort of telomere end protection as it allows to regularly add telomeric DNA sequences to the eroding telomeres of dividing cells [5]. Extensive studies on telomeric proteins from various organisms, starting in the 1990s, led to the notion that the association of these telomeric proteins with telomeric DNA provides efficient means for masking these natural DNA double-strand breaks (DSBs) from inappropriate repair.

To date, in various organisms, a number of telomeric proteins have been implicated in preventing access of various DNA modification proteins to the telomeres. In the budding yeast Saccharomyces cerevisiae, Rap1, which binds telomeric double-stranded DNA repeats, inhibits both telomerase-dependent telomere elongation and telomere fusions by NHEJ [6-8]. The Rap1-Rif1-Rif2 complex in fact functionally resembles shelterin, the telomere end protection complex in mammals [9]. The Yku complex, which plays a major role in NHEJ, also localizes at the telomeres, at the junction between the double-stranded and single-stranded DNA (ssDNA) repeats where it functions in telomere end protection [10]. Finally, another major component for telomere capping in $S$. cerevisiae is a heterotrimeric complex composed of Cdc13, Stn1 and Ten1 that binds the single-stranded overhangs of the telomeres [11-14]. Recent work has shown that Rif2 levels are reduced at short telomeres and that this is important for the recruitment of Mre11-Rad50-Xrs2 (MRX) and of Tel1 at these telomeres [15], an event leading to the recruitment of telomerase for re-elongation [1620]. By favoring telomerase recruitment, the reduction of Rif2 levels may therefore help distinguish short telomeres from DSBs [15]. In the same study, at short telomeres, Mec1 and Replication Protein A (RPA) levels were found to be strongly reduced compared to their levels at DSBs, thus providing a way to prevent putative DNA damage signaling and DNA repair attempts [15].

RPA is a major, evolutionary conserved, ssDNA-binding complex that is essential for numerous transactions during DNA synthesis and DNA damage signaling and repair [21-24] everywhere in the genome including, in the budding yeast $S$. cerevisiae, at the telomeres [25]. The unique function of RPA in DNA damage signaling identified to date, in both humans and S. cerevisiae, is to recruit the ATR/ATRIP and Mec1-Ddc2 module of the DNA damage 
checkpoint machinery, respectively [26]. Under normal circumstances, in cells with undamaged telomeres, Cdc13 prevents Mec1 activation [11] and, presumably therefore, RPA recruitment. However, it is not known yet whether Cdc13 acts primarily through limiting end resection or whether it also blocks, by competition, RPA binding to the telomeric ssDNA $[4,14]$. In humans, the interactions between POT1 and RPA have been better documented, with the recent discovery of a TERRA- and hnRNPs-based mechanism that can displace RPA, but not POT1, activity $[27,28]$.

It is crucial to fully understand the relationships between telomere uncapping and cell cycle progression as efforts to uncover the underlying mechanisms driving genome instability in cancer cells have revealed a prominent role for telomeres [29]. Telomere uncapping is not an unusual fate as, for instance, the numerous events leading to temomerase recruitment at the telomeres may provoke transient but necessary periods of loss of telomere end protection. DNA replication itself is susceptible to create telomeric damage resulting from pausing or even stalling of the replication forks in the telomeric regions due to the repetitive nature of the DNA sequences [30-32]. In the present study, to better understand the relationships between telomere uncapping and cell cycle progression we analyzed mutants of $S$. cerevisiae RPA, previously described [33], in a genetic context that has been extensively used as a mean to provoke telomere uncapping, namely the temperaturesensitive cdc13-1 mutation [11].

\section{Experimental procedures}

\subsection{Yeast strains, viability assays and Western blotting}

Yeast strains used in this study were derivatives of BF264-15Daub (ade1 his2 leu2-3,112 trp1-1a ura3Dns), as described previously [12]. Yeast cultures were grown at the indicated temperatures in YEP (1\% yeast extract, $2 \%$ bacto-peptone, $0.005 \%$ adenine, $0.005 \%$ uracile) supplemented with $2 \%$ glucose (YEPD), sucrose or galactose, or in selective minimal medium. All strains were made isogenic by back crossing at least five times against our genetic background. Strain origins, prior to back crossing, were as described previously [34].

Construction of the rfa1 mutants analyzed here has been described previously [33]. Activation of the checkpoint kinase Rad53 was assessed by Western blotting as described previously [33].

The viability of cells previously grown in liquid was determined by performing and analyzing the so-called "drop tests" or "spot assays". To do this, cells from exponential growth cultures were counted with a hematocytometer and the cultures were then serially diluted by $1 / 5^{\text {th }}$ or $1 / 10^{\text {th }}$ and spotted onto either selective plates or YEPD non-selective plates, as required, and incubated at the desired temperature for 2-3 days before being photographed. 


\subsection{Telomere length measurement, telomere organization and detection of ssDNA}

Assessment of telomere length and telomere organization in cells with either non recombining or recombining telomeres was done as described previously [12]. Briefly, genomic DNAs were prepared, separated in a $0.9 \%$ agarose gel (in TBE) run in TBE buffer overnight and, after denaturation, transferred and hybridized with a 270 base pair $\mathrm{TG}_{1-3}{ }^{32} \mathrm{P}$ labeled telomeric probe as described previously [12]. Following digestion of genomic DNA with Xhol, to cut within the $Y^{\prime}$ regions of chromosomes, telomere tracts of wild-type cells appear as a broad band of $\sim 1.1-1.4 \mathrm{~Kb}$ which represents the average length of most chromosomes. Telomeric recombination was assessed as previously described [37]. Detection of ssDNA at the telomeres was performed as described previously [13,34]. Results were analyzed using an FLA-5100 Fuji phosphorimager or a GE Storm phosphorimager and the ImageGauge software.

\section{Results}

3.1. rfa1 mutants defective in telomeric damage-induced cell cycle progression independently of the DNA damage checkpoint

Temperature-sensitive cdc13-1 mutant cells of Saccharomyces cerevisiae exhibit uncapped telomeres under the form of abnormally high levels of ssDNA in both telomeric and subtelomeric regions of the chromosomes [11]. There is both genetic and biochemical evidence that RPA is recruited at cdc13-1-induced telomeric DNA damage sites [25,26]. Yet, although RPA recruited at DSBs mediates repair by homologous recombination [35], the cdc13-1 cells do not trigger recombination or any other apparent signs of repair. In agreement with this view, cdc13-1 cells experiencing telomeric recombination due to inactivation of telomerase could now grow at the semi-permissive temperature of $29^{\circ} \mathrm{C}$ (Figure 1A, B). Presumably, in these cells telomeric recombination provided some sort of DNA repair, as observed in post-senescence survivors from telomerase-negative cells [36]. It has been previously established that the slow growth of $c d c 13-1$ cells at $29^{\circ} \mathrm{C}$, as well as the total arrest of proliferation at temperatures above $32{ }^{\circ} \mathrm{C}$, was due to activation of the Mec1Rad53-Mec3 DNA damage checkpoint network, which induces a block to cell cycle progression [11]. Thus, as shown by Hartwell and colleagues [11] and subsequently by several other laboratories, telomerase-positive cdc13-1 cells in which the DNA damage checkpoint had been inactivated exhibited improved growth at $29^{\circ} \mathrm{C}$ (Figure 1C; override of the arrest by the rad24 $\Delta$ mutation; compare rows 1 and 2; Rad24 is a component of an RFClike complex that is essential for loading the Mec3-Ddc1-Rad17 checkpoint complex).

We previously isolated rfa1 mutants that, in a telomerase-negative background, were specifically deficient in type I telomeric recombination on the $Y^{\prime}$ subtelomeric sequences (but 
not in type II recombination on the $\mathrm{TG}_{1-3}$ repeats) but proficient in activating the DNA damage checkpoint [33]. In the current study, we analyzed these rfa1 mutants (see mutations in Table 1) in a cdc13-1 background. The rfa1-1, -9, -10, -11, and -12 mutants rescued cdc13-1 at $29^{\circ} \mathrm{C}$ in a way similar (although to different extents) to the checkpoint-deficient mutants rfa1-t11 and rad24L (Figure 1C; note that rfa1-12 was not deficient in type I recombination but was used, in the previous study, as a negative control; ref. 33). rfa1-t11, first described in ref. 35, was later shown, in the cdc13-1 background, to be checkpoint-deficient [25], the mutant protein being defective in recruiting the Mec1-Ddc2 checkpoint complex [26]. Therefore the rfa1-t11 and rad24 $\Delta$ mutations each impinge on one of two parallel pathways of the same checkpoint network, thereby explaining their similar phenotypes upon cdc13-1induced damage (Figure 1C). Surprisingly, the rfa1 mutants from our previous study [33] analyzed here, although failing to arrest upon damage (Figure 1C; compare rows 1, 2 and 37), were still proficient in activating the DNA damage checkpoint, as judged by full activation of Rad53, a pivotal protein kinase essential for this checkpoint (Figure 2A), thus confirming our previous analysis [33]. To further document this apparent contradiction, we set out to genetically inactivate a component of the DNA damage checkpoint pathway in the cdc13-1 rfa1 double mutants. The rationale for these experiments was that simultaneously inactivating two checkpoint genes in the same DNA damage response pathway does not provoke a more severe checkpoint defect than inactivating either one of these two same genes (see, for instance, ref.37,38). Combining an rfa1 mutation with a mutation in a gene of the checkpoint network, here $M E C 3$, resulted in a clear additional effect in the cdc13-1 background, an effect that was best visible at $30^{\circ} \mathrm{C}$ (Figure 2B).

Simple observation of the checkpoint-deficient cdc13-1 rad24 $\Delta$ and cdc13-1 rfa1 mutants under the light microscope evidenced two different types of arrest existing (Figure 2C). In seminal papers that led to the discovery of a DNA damage checkpoint pathway in all eukaryotic cells, Hartwell and colleagues observed that checkpoint-negative cdc13-1 double mutants failed to arrest in the G2 phase and died rapidly after a few cell divisions following shift to the restrictive temperature $[37,39,40]$. After $2 \mathrm{~h}$ at $34^{\circ} \mathrm{C}, \mathrm{cdc} 13-1 \mathrm{rad} 24 \Delta$ mutant cells indeed exhibited the behavior previously described by these authors, while the cdc13-1 rfa19 mutant did not, and, instead, arrested in the G2 phase very much like the checkpointproficient cdc13-1 mutant (Figure 2C). This strongly suggested that the mechanisms deregulated in the rfa1 mutant are not the same as those deregulated in the classical checkpoint mutant.

It is important to note that checkpoint deficiency leading to improved growth is specific for the cdc13-1 mutation and is usually explained by the fact that, at semi-permissive temperatures for growth, the telomeric DNA damage is not too severe and can be tolerated 
through many rounds of cell divisions $[11,37,41]$. However, this is different in a large number of other DNA-damaging backgrounds tested, such as the temperature-sensitive cdc17-1 mutant, in which abrogation of the checkpoint results in a decrease in cell viability as temperature increases [37]; $C D C 17$ codes for the catalytic subunit of the DNA polymerase alpha-primase complex; ref. 42,43). Interestingly, while the ddc1s mutation and the rfa1-t11 mutations, both abrogating the DNA-damage checkpoint, decreased cdc17-1 cell survival at the semi-permissive temperature of $32^{\circ} \mathrm{C}$, the rfa1-12 mutation had no effect, further suggesting that this mutant is not deficient in DNA-damage checkpoint (Figure 2D).

In summary, by biochemical (Fig. 2A), genetic (Fig. 2B, D) and morphological (Fig. 2C) criteria, the data above established that upon cdc13-1-induced telomeric DNA damage the rfa1-1, $-9,-10$ and -12 mutations, although provoking a deregulation in cell cycle arrest apparently similar to that in DNA damage checkpoint mutants (Figure 1C), nevertheless did not impinge on the same targets for controlling cell cycle progression.

\subsection{Rescue of cdc13-1 by rfa1-1, $-9,-10$, or -12 is not due to a decrease in DNA damage} The 5' to 3' double-stranded DNA EXO1 exonuclease is the main exonuclease -and only one identified to date- responsible for telomeric CA strand digestion [44-46], creating the long TG ssDNA overhang in cdc13-1 cells at restrictive temperature that is at least part of the damage sensed by the DNA-damage checkpoint [11]. Potentially, the rfa1 mutants analyzed here could rescue cdc13-1 growth by preventing access of Exo1 to telomeric DNA, thereby decreasing the amount of damaged telomeric DNA. As expected from previous results [44], deleting EXO1 partially rescued cdc13-1 cells at $29^{\circ} \mathrm{C}$ (Figure $3 \mathrm{~A}$, compare rows 1 and 6 ), to the same extent as the rfa1 mutants and the DNA-damage checkpoint mutants. We reasoned that if the Rfa1 mutant protein acted through Exo1 to rescue cdc13-1 (in other words if the rfa1 mutation led to inactivation of Exo1), then the triple cdc13-1 rfa1 exo1s mutant would grow like the double cdc13-1 rfa1 and the cdc13-1 exo1s double mutants. However, on the contrary, we observed that the triple cdc13-1 rfa1 exo1 $\Delta$ mutants grew better than the two corresponding double mutants (Figure 3A, compare rows 7-10 to row 6). These data strongly suggested that in the rescue of cdc13-1 these rfa1 mutations were not acting by preventing Exo1 access to telomeric DNA.

To confirm the data above by a distinct approach, we prepared genomic DNAs from the cdc13-1, cdc13-1 mec3s, cdc13-1 rfa1-t11, cdc13-1 exo1s and cdc13-1 rfa1 mutants and set out to detect telomeric ssDNA in these cells after growth at the restrictive temperature of $34^{\circ} \mathrm{C}$ by applying native conditions of hybridization to the telomeric probe. For the denatured control gel, the genomic DNA was digested with EcoRI instead of the more commonly used Xhol enzyme, because the EcoRI site is more centromere-proximal than the 
Xhol site, releasing telomeric fragment of $\sim 4.0 \mathrm{~Kb}$ instead of $\sim 1.2 \mathrm{~Kb}$ for Xhol. Therefore, the $E c o R I$ site is far enough from the telomeric ssDNA for the enzyme to cut efficiently. On the native gel, the Xhol-digested DNA displayed the classical smear characteristic of abnormal accumulation of telomeric ssDNA induced by the cdc13-1 mutation [11] in all

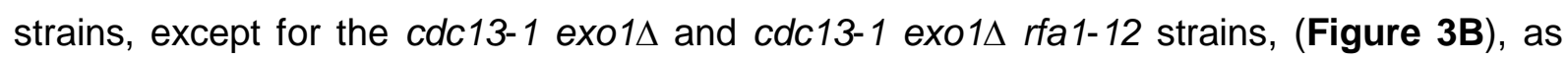
expected from the genetic data of Figure $3 A$. Therefore, these rfa1 mutants do not prevent telomeric degradation by Exo1 and, more generally, do not appear to lead to a diminution of cdc13-1-induced telomeric DNA damage.

3.3 The rfa1-12 mutation can trigger immediate telomeric recombination at uncapped telomeres

As explained above and illustrated in Figure $1 \mathrm{~A}, \mathrm{~B}$, telomeric recombination can repair the cdc13-1-induced telomeric damage. Inferring that the rescue of $c d c 13-1$ by the analyzed rfa1 mutants could possibly be due to recombination-dependent telomere repair, we looked at the structure of telomeres in these strains by Southern blot. Telomeres of otherwise wild-type cells were normal in the rfa1 mutants (Figure 4A, left lanes). In the cdc13-1 background and at the permissive temperature of $24^{\circ} \mathrm{C}$, telomeres tended to be shorter than those in the wild type (with the exception of cdc13-1 rfa1-1), but still homogenous in length (Figure 4A, right lanes). We then grew the cdc13-1 rfa1 mutants at semi-permissive temperature $\left(29^{\circ} \mathrm{C}\right)$ for several generations to give the telomeres time to be processed by the DNA repair activities induced by the cdc13-1 damage. In this experiment, control mutant strains also capable of growth at semi-permissive temperature, such as cdc13-1 mec3s (checkpoint-deficient; [37], cdc13-1 bmh1 147 ] and cdc13-1 exo1s [44] were included. After reaching equilibrium at $29^{\circ} \mathrm{C}$, telomeres in the cdc13-1 rfa1-1, -9 and -10 mutants were of various length depending on the mutation, but without excessive lengthening or shortening (Figure 4B) and somewhat more heterogeneous in length than at $24^{\circ} \mathrm{C}$ (compare Figure $4 \mathrm{~A}$ right lanes and Figure 4B). At $29^{\circ} \mathrm{C}$, the telomeres in cdc13-1 rfa1-1 tended to be longer than in the wild type and those in both the cdc13-1 rfa1-9 and in cdc13-1 rfa1-10 tended to be shorter than in the wild type, just like at $24^{\circ} \mathrm{C}$ (Figure $\left.4 \mathrm{~A}\right)$.

In sharp contrast with the other three mutants, the cdc13-1 rfa1-12 mutant exhibited clear disorganization of telomere organization, much more dramatic than simple variations in length or homogeneity, which, interestingly, resembled a classical profile of type II recombination (Figure 4B; ref. 48-50). Data presented below indeed established that telomeric recombination takes place in the cdc13-1 rfa1-12 mutant at $29^{\circ} \mathrm{C}$.

3.4 Improved growth in cdc13-1 rfa1-12 can take place in the absence of telomeric recombination 
We were intrigued by the fact that the rfa1-1, rfa1-9 and rfa1-10 mutants also exhibited improved growth at $29^{\circ} \mathrm{C}$ in the cdc13-1 background even though their telomeres were not recombining, as shown above in Figure 4B. We therefore next asked whether the rescue of cdc13-1 growth by rfa1-12 was indeed caused by telomeric recombination, as can be found under other circumstances (Figure 1A, B). Type II telomeric recombination depends on Rad52, a protein essential for basically all types of recombination, and the Mre11-Rad50Xrs2 (MRX) complex [36,48,49]. A diploid bearing the desired heterozygous mutations, CDC13/cdc13-1 RFA1/rfa1-12 RAD52/rad52A, was induced to sporulate and the drop testbased growth assays of the strains with the desired genotype performed as soon as possible after spore selection. After 3 days of growth following spore selection, rescue of $c d c 13-1$ by the rfa $1-12$ mutation at $27.5^{\circ} \mathrm{C}$ could be readily observed (Figure 5A, compare rows 1 and 3). Most importantly, this rescue was not inhibited by the absence of Rad52 (Figure 5A, compare rows 3 and 4). We prepared genomic DNAs from these cells at day 3 (corresponding to the time at which the drop tests of Figure 5A were completed, day 0 being the time when the spore selection process was completed) and, in parallel, let these cells grow for 2 and 4 more days at $27.5^{\circ} \mathrm{C}$ before preparing their genomic DNAs for telomere structure analysis by Southern blot (Figure 5B). As expected, after 3 days of growth (day 3 defined above) the cdc13-1 rfa1-12 cells did not exhibit a recombining telomeric profile (Figure 5B, day 3). It was only at day 7 that the presence of a large smear of telomeric DNA attested that recombination had started (Figure 5B, day 7, left lane). Actual recombination in the cdc13-1 rfa1-12 cells was confirmed by the disappearance of that smear when RAD52 had been deleted (Figure 5B, day 7, right lane). Therefore, at early times following strain construction the cdc13-1 rfa1-12 cells grew at $27.5^{\circ} \mathrm{C}$ (Figure 5A) while their telomeres had not yet started to recombine, as attested by Southern analysis (Figure 5B). In summary,

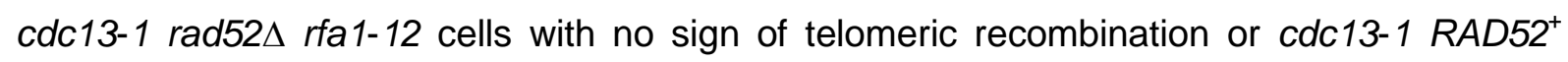
rfa1-12 cells prior to initiation of telomeric recombination, at day 3 , nevertheless exhibited deregulated growth upon telomere uncapping, leaving us with the possibility for other mechanism being deregulated in the rfa1 mutant.

\subsection{Improved growth in cdc13-1 rfa1 requires Tel1 kinase activity as well as Est2/telomerase} Following thorough analyses of genetic interactions, in a cdc13-1 background, between the rfa1 mutations studied here and mutations in genes previously implicated in telomeric pathways, we observed that, at semi-permissive temperatures for growth for cdc13-1, inactivation of TEL1 partially suppressed cdc13-1 rescue by rfa1-1 and totally suppressed that by rfa1-12 (Figure 6A). To know whether the suppression of cdc13-1 rfa1 rescue by Tel1 required its kinase activity, we modified the wild-type TEL1 chromosomal locus for expression of a tel1-kd mutation [51]. Inhibition of Tel1 kinase activity inhibited the rescue of 
cdc13-1 by all rfa1 mutants to various extents, the effect being stronger for rfa1-12 (Figure 6B, top panels). The absence of a synthetic defect between the tel1-kd and rfa1 mutants in an otherwise wild-type background suggested that the genetic interactions observed between the rfa1 and te/1 mutations are linked to telomere protection and not to some other more general effect (Figure 6B, bottom panel).

Because Tel1 has been shown to mediate telomere elongation by telomerase at short telomeres, a reaction that appears to involve the initial recruitment of MRX at these ends followed by transient association with Tel1 [17-19], we performed experiments using the est2-DF338 mutation, which displays short telomeres, decreased telomerase activity and decreased binding to TLC1 [52]. The est2-DF338 mutation exhibited only slight synthetic growth defects in combination with cdc13-1 (Figure 6D, bottom left panel, compare rows 2 and 3 , at $24^{\circ} \mathrm{C}$ ). The short telomere phenotype conferred by the est2-DF338 mutation in different backgrounds is shown in Figure 6C. When combined with the cdc13-1 rfa1 mutations, the est2-DF338 mutation suppressed the rescue of $c d c 13-1$ by the rfa1 mutations (Figure 6D).

We tested the effects of the mre11 and yku70 null mutations on the growth characteristics of the cdc13-1 rfa1 mutants. However, since both mre11 $1 \mathrm{~s}$ and $y k u 70 \Delta$ exacerbated the growth defects conferred by $c d c 13-1$, as previously reported by several other laboratories, this rendered the interpretation of the drop test-based growth assays of the strains difficult (data not shown).

3.6 Telomere elongation in telomerase-negative cells does not confer cdc13-1 rfa1-12 improved growth

One possible interpretation of the data above was that telomerase-mediated telomere elongation might be responsible for the deregulated growth of these rfa1 mutants upon telomere uncapping. To further challenge this hypothesis, we reasoned that it would be interesting to find a way to achieve the same goal as that resulting from telomerase functioning, namely synthesizing telomere repeats, but in the absence of telomerase. In budding yeast, an alternative pathway for adding $\mathrm{TG}_{1-3}$ repeats at the ends of telomeres is through the so-called type II pathway of telomeric recombination that takes place in telomerase-negative post-senescence survivors [36,49]. Type I survivors can also be generated in these mutants but they amplify the sub-telomeric repeats rather than the $\mathrm{TG}_{1-3}$ repeats [49]. In fact, the cdc13-1 background induces 100\% type II [53]. We constructed several cdc13-1 t/c1 $1 \Delta$ rfa1 strains as well as the cdc13-1 t/c1 $R F A 1^{+}$as a control (TLC1 encodes the telomerase RNA subunit) and let them grow for over 100 generations, past the time required for the cells to enter telomeric senescence, around 75 generations. As expected, telomeric recombination bypassed senescence in a minority of cells and post- 
senescence survivors were generated [36,54], of type II in the present situation. As shown in Figure 7, both the cdc13-1 t/c1s rfa1 and cdc13-1 t/c1 $R F A 1^{+}$cells could grow at $29^{\circ} \mathrm{C}$. This was expected as telomeric recombination rescues the cdc13-1 mutation as shown in Figure 1A. However, interestingly, the rfa1 mutant cells did not grow better than the $R F A 1^{+}$ cells, indicating that in the absence of telomerase the rfa1 mutations no longer confer growth advantage (Figure 7). To make sure that some aspect of telomerase activation was not taking place in the absence of TLC1 via the Est2 catalytic subunit, we also performed the analysis by simultaneously introducing the est2-DF338 mutation and obtained the same result ((Figure 7). Therefore, the rfa1 mutations no longer confer cdc13-1 cells a growth advantage in the absence of functional telomerase.

\section{Discussion}

The present work reveals two novel important functions for $S$. cerevisiae RPA, one in protection against inappropriate telomere recombination, the second one in preventing, upon telomere uncapping, cell proliferation by a checkpoint-independent pathway.

S. cerevisiae RPA has been shown to assume a function in telomere length control and telomerase recruitment [55-57]. However, to our knowledge, RPA has never been shown before to play a role in preventing telomeric recombination. The interpretation of our data (Fig. 4) attesting to a role of RPA in telomere end protection is straightforward. Indeed, as shown previously for other telomere end protection proteins, notably Cdc13 and the Yku complex [14], in the presence of telomere damage, the Rfa1-12 (L227S) protein triggered type II recombination. Note that the nature of telomere uncapping provoked here, namely the temperature-sensitive cdc13-1 mutation, has been previously shown to produce 100\% type II recombination [53]. Therefore, it is not known yet whether RPA also protects subtelomeric sequences from undergoing inappropriate recombination. In general, in budding yeast, two situations have been reported to be associated with the triggering of telomeric recombination and the preceding announcing events, namely telomeric senescence. The more frequent one is the combination of mutations in two telomere end protection proteins, such as cdc13-1 and $y k u 70 \Delta$ for instance [58]. It is not currently known whether the rfa1-12 mutation by itself creates telomeric damage. The second situation that was found to associate with telomere recombination was when loss of telomere capping, again provoked by the cdc13-1 mutation, was accompanied by inactivation of the G2/M DNA damage checkpoint [34]. Although the rfa1-12 mutation does not confer a defect in that checkpoint, its implication in a parallel pathway of cell cycle control, as documented in the present study, makes the scenario observed with cdc13-1 mec3s mutants, for instance [34] also likely to happen with cdc13-1 rfa1-12. 
In view of the present data, RPA appears to represent a previously unsuspected key factor capable of operating a switch between telomerase-based telomere maintenance and recombination-based telomere maintenance, also known as the ALT pathway. Recent data obtained in mice have suggested that the system of telomere maintenance present at a given time, telomerase or ALT, might represent an unstable equilibrium that can easily shift from one system to the other when certain proteins, for instance the RTEL1 helicase, the DNA damage checkpoints or telomerase itself have been compromised $[59,60]$. At present, there is no evidence that RPA can play major roles in tumor initiation or progression as a result from telomeric disturbances. In fact, to our knowledge, mutations in RPA have not been directly associated with cancer, with the exception of a report that mice engineered to heterozygously express RPA1 L221P displayed elevated rates of cancer [61]. This was probably due to the L221P mutation conferring defects in checkpoints and homologous recombination, as seen in yeast with the equivalent mutation [62].

The present data represent further analysis of previously described rfa1 mutants, rfa1-1, rfa1-9, rfa1-10, rfa1-11 and rfa1-12 [33]. Importantly for the comprehension of the present work, these mutations were shown to be resistant to methylmethane sulfonate treatment, like the wild type but unlike $d d c 1 \Delta$ and $\operatorname{rad51\Delta }$, and were therefore presumed to be proficient in DNA repair [33]. Here, these mutants have been shown to confer deregulation of the cell cycle arrest that is normally triggered upon telomere uncapping, provoked by the temperature-sensitive cdc13-1 mutation. This should not have been surprising, since a similar phenotype had already been reported concerning the rfa1-t11 mutant ([25]; see also Fig. 1C). Suppression of cdc13-1 arrest by the rfa1-t11 mutation has been shown to be due to an inability for the Rfa1-t11 protein to recruit the Mec1-Ddc2 checkpoint complex [26]. However, suppression of cdc13-1 arrest by the rfa1 mutations analyzed here was nevertheless surprising since these mutants were previously reported to be checkpoint proficient, in this same genetic background [33], as we confirmed here (Fig. 2A). By genetics, we further demonstrated here that these Rfa1 mutant proteins acted in a separate pathway than that controlled by the Mec1-Rad53-Mec3 DNA damage checkpoint (Fig. 2B). In fact, this was obvious when examining the morphology of the mutant cells of the RPA pathway and comparing with that of the mutant cells of the checkpoint pathway (Fig. 2C). Assessment of telomeric damage strongly suggested that these cdc13-1 rfa1 mutants did not accumulate less damage than the cdc13-1 RFA1+ mutant, which could have explained their improved growth, and did not impinge on the Exo1 pathway of damage generation (Fig. 3).

Initially, to try to explain the checkpoint-independent override of the cell cycle progression in the cdc13-1 rfa1 mutants, we reasoned that cell cycle control proteins should be suspected of being involved. In $S$. cerevisiae, besides with Mec1-Ddc2, RPA has never 
been implicated in physical or functional interactions with proteins of control of the cell cycle, with the notable exception of the report of a physical interaction between Rfa1 and Cdc20 by affinity capture mass spectrometry in a high-throughput study [63]. We have investigated that possibility but have been so far unable to confirm this interaction by two hybrid or to recapitulate it by in vivo co-immunoprecipitation between chromosomally expressed $\mathrm{Myc}_{2}$ Cdc20 and $\mathrm{HA}_{2}$-Rfa1 (Grandin and Charbonneau, unpublished results).

In parallel, a thorough genetic analysis using combinations of the rfa1 mutations analyzed here and mutations in proteins involved in telomeric pathways led us to the surprising conclusion that deregulation of the cell cycle arrest in the cdc13-1 rfa1 mutants could be strongly suppressed by inactivating either TEL1 or EST2 (Fig. 6). Mre11 and Tel1 cooperate in the recruitment of telomerase at short telomeres [17-20]. It is therefore reasonable to conclude that either a lack of telomerase loading (directly through the est2DF338 mutation or indirectly through the tel1 mutation) or shorter than normal telomeres could lead to suppression of checkpoint-independent cell cycle control deregulation in cdc131 rfa1. Unfortunately, we have been unable to rigorously test the effects of mutations in the $\mathrm{MRX}$ and Yku complexes due to synthetic growth defects with cdc13-1. Additional experiments in telomerase-negative post-senescence survivors (Fig. 7) indicated that the requirement of the rfa1 phenotype (deregulation of cell cycle control upon damage) for telomeric repeats addition by telomerase could not be mimicked by addition of these repeats by telomere recombination. We propose that wild-type RPA normally prevents cell cycle progression upon telomere uncapping by two parallel pathways. One is the already described pathway of recruitment of an essential module of the DNA damage checkpoint machinery, Mec1-Ddc2 [26]. The second one might be to block telomerase action in order to prevent further addition of telomeric repeats. Addition of telomeric repeats can provide healing, for instance at DSBs, a reaction that is normally prevented by specific proteins, such as Pif1 [64]. However, in the present situation, potential healing by telomere repeat addition would not prevent telomere uncapping in the long term, the damage being persistent due to the continuous application of the cdc13-1-mediated insult. In fact, we have seen that the telomeric damage is not less in the rfa1 mutants, at least at $34^{\circ} \mathrm{C}$ (Fig. 3B). However, it is possible that unscheduled functioning of telomerase at intervals simultaneously at one or more uncapped telomeres could give the cells a sort of short respite from ongoing damage, respite that would be immediately followed by insult, thus undetectable at least with the sensitivity allowed by our measurements. In the cdc13-1 rfa1 mutants, such short bursts of telomeric repeats addition might confer regular periods of relief allowing in the end improved cell proliferation compared with the $R F A 1^{+}$counterpart.

Particularly noteworthy are the F238 and F269 mutations found in rfa1-11 and rfa110 , respectively, that are conserved aromatic residues that make direct contact with ssDNA 
[65]. These mutations may alter the ssDNA binding properties and affinity of RPA, its most important and fundamental attribute, and influence the telomeric pathways in which RPA is involved. In damaged cells, ill-positioned mutant RPA might give inappropriate access to Tel1. By blocking access of Tel1 at uncapped telomeres, wild type RPA might assume a function of marking the telomere for repair rather than for elongation by telomerase. Alternatively, RPA might directly bind telomerase to prevent it from being active in the presence of telomeric damage. This hypothesis is particularly interesting given the recently described physical interaction between RPA and telomerase in budding yeast [57]. We have been unable to test this hypothesis as we could not detect physical interaction between chromosomally expressed $\mathrm{Myc}_{18}$-Est2 and $\mathrm{HA}_{2}$-Rfa1 or $\mathrm{HA}_{2}$-Rfa1-12 (Grandin and Charbonneau, unpublished results). Because we clearly detect each of these proteins upon immunoprecipitation but not attached to each other neither at normal or uncapped telomeres (Grandin and Charbonneau, unpublished results), we believe that a putative RPA-telomerase physical interaction is not key in underlining the deregulation of the rfa1 mutants described here.

At the telomeres, RPA is physically present next to other ssDNA-binding proteins, such as Cdc13 and POT1, and assumes important roles in order to allow the coordinated completion of both telomere replication and telomere end protection [28]. The present data on the role of RPA in telomere end protection in S. cerevisiae will hopefully contribute to the elucidation of these pathways.

\section{Conflict of interest statement}

The authors declare that there are no conflicts of interest.

\section{Acknowledgements}

We are grateful to Akira Matsuura for the gift of tel1-kd plasmid, Neal Lue for the gift of the est2-DF338 plasmid, Andrew Emili for the gift of the RAD53-HA $\mathrm{A}_{2}$ plasmid, Thomas Petes for the gift of the tel1::URA3 plasmid and Daniel Gottschling for the gift of the t/c1::URA3 plasmid. We also thank the "Département Génomique PPF ASB" facility at University François Rabelais of Tours for access to the Storm phosphorimager. This work was «supported by grants from the "Centre National de la Recherche Scientifique to UMR 5239/Ecole Normale Supérieure de Lyon" and to "UMR 7292/Université François Rabelais de Tours, France“.

\section{References}

[1] H.J. Muller, The remaking of chromosomes. Collecting Net. 13 (1938) 181-195. 
[2] B. McClintock, The fusion of broken ends of chromosomes following nuclear fusion. Proc. Natl. Acad. Sci. USA 28 (1942) 458-463.

[3] M.P. Longhese, DNA damage response at functional and dysfunctional telomeres. Genes Dev. 22 (2008) 125-140.

(4]. T. de Lange, How telomeres solve the end-protection problem. Science 326 (2009) 948952.

[5]. E. Gilson, V. Géli, How telomeres are replicated. Nat. Rev. Mol. Cell. Biol. 8 (2007) 825838.

[6] S. Marcand, E. Gilson, D. Shore, A protein-counting mechanism for telomere length regulation in yeast. Science 27 (1997) 986-990.

[7] B. Pardo, S. Marcand, Rap1 prevents telomere fusions by nonhomologous end joining. EMBO J. 24 (2005) 3117-3127.

[8] S. Marcand, B. Pardo, A. Gratias, S. Cahun,, I. Callebaut, Multiple pathways inhibit NHEJ at telomeres. Genes Dev. 22 (2008) 1153-1158.

[9] W. Palm, T. de Lange, How shelterin protects mammalian telomeres. Annu. Rev. Genet. 42 (2008) 301-334.

[10] S. Gravel, M. Larrivée, P. Labrecque, R.J. Wellinger, Yeast Ku as a regulator of chromosomal DNA end structure. Science 280 (1998) 741-744.

[11] B. Garvik, M. Carson, L. Hartwell, Single-stranded DNA arising at telomeres in cdc13 mutants may constitute a specific signal for the RAD9 checkpoint. Mol. Cell. Biol. 15 (1995) 6128-6138.

[12] N. Grandin, S.I. Reed, M. Charbonneau, Stn1, a new Saccharomyces cerevisiae protein, is implicated in telomere size regulation in association with Cdc13. Genes Dev. 11 (1997) 512-527.

[13] N. Grandin, C. Damon, M. Charbonneau, Ten1 functions in telomere end protection and length regulation in association with Stn1 and Cdc13. EMBO J. 20 (2001) 1173-1183.

[14] D. Shore, A. Bianchi, Telomere length regulation: coupling DNA end processing to feedback regulation of telomerase. EMBO J. 28 (2009) 2309-2322.

[15] J.S. McGee, J.A. Phillips, A. Chan, M. Sabourin, K.Paeschke, V.A. Zakian, Reduced Rif2 and lack of Mec1 target short telomeres for elongation rather than double-strand break repair. Nat. Struct. Mol. Biol. 17 (2010) 1438-1445.

[16] A. Bianchi,A. D. Shore, Increased association of telomerase with short telomeres. Genes Dev. 21 (2007) 1726-1730.

[17] M. Chang, M. Arneric, J. Lingner, Telomerase repeat addition processivity is increased at critically short telomeres in a Tel1-dependent manner in Saccharomyces cerevisiae. Genes Dev, 21 (2007) 2485-2494. 
[18] R.E. Hector, R.L. Shtofman, A. Ray, B.R. Chen, T. Nyun, K.L. Berkner, K.W. Runge, Tel1p preferentially associates with short telomeres to stimulate their elongation. Mol. Cell 27 (2007) 851-858.

[19] M. Sabourin, C.T. Tuzon, V.A. Zakian, Telomerase and Tel1p preferentially associate with short telomeres in S. cerevisiae. Mol. Cell 27 (2007) 550-561.

[20] V. Viscardi, D. Bonetti, H. Cartagena-Lirola, G. Lucchini, M.P. Longhese, MRXdependent DNA damage response to short telomeres. Mol. Biol. Cell 18 (2007) 3047-3058 [21] S.K. Binz, A.M. Sheehan, M.S. Wold, Replication protein A phosphorylation and the cellular response to DNA damage. DNA Rep. 3 (2004) 1015-1024.

[22] K. Sakaguchi, T. Ishibashi, Y. Uchiyama, K. Iwabata, The multi-replication protein A (RPA) system--a new perspective. FEBS J. 276 (2009) 943-963.

[23] S. Broderick, K. Rehmet, C. Concannon, H.P. Nasheuer, Eukaryotic single-stranded DNA binding proteins: central factors in genome stability. Subcell. Biochem. 50 (2010) 143163.

[24] R.L. Flynn, L. Zou, Oligonucleotide/oligosaccharide-binding fold proteins: a growing family of genome guardians. Crit. Rev. Biochem. Mol. Biol. 45 (2010) 266-275.

[25] H.S. Kim, S.J. Brill, Rfc4 interacts with Rpa1 and is required for both DNA replication and DNA damage checkpoints in Saccharomyces cerevisisae. Mol. Cell. Biol. 21 (2001) 3725-3737.

[26] L. Zou, S.J. Elledge, Sensing DNA damage through ATRIP recognition of RPA-ssDNA complexes. Science 300 (2003) 1542-1548.

[27] R.L. Flynn, R.C. Centore, R.J. O'Sullivan, R. Rai, A. Tse, Z. Songyang, S. Chang, J. Karlseder, L. Zou, TERRA and hnRNPA1 orchestrate an RPA-to-POT1 switch on telomeric single-stranded DNA. Nature 471 (2011) 532-536.

[28] R.L. Flynn, S. Chang, L. Zou, RPA and POT1: friends or foes at telomeres? Cell Cycle 11 (2012) 652-657.

[29] S.E. Artandi, R.A. DePinho, Telomeres and telomerase in cancer. Carcinogenesis 31 (2010) 9-18.

[30] A.S. Ivessa, J.Q. Zhou, V.P. Schulz, E.K. Monson, V.A. Zakian, Saccharomyces Rrm3p, a 5' to 3' DNA helicase that promotes replication fork progression through telomeric and subtelomeric DNA. Genes Dev. 16 (2002) 1383-1396.

[31] S. Makovets, I. Herskowitz, E.H. Blackburn, Anatomy and dynamics of DNA replication fork movement in yeast telomeric regions. Mol. Cell. Biol. 24 (2004) 4019-4031.

[32] N. Fouché, S. Özgür, D. Roy, J.D. Griffith, Replication fork regression in repetitive DNAs. Nucleic Acids Res. 34 (2006) 6044-6050. 
[33] N. Grandin, M. Charbonneau, Control of the yeast telomeric senescence survival pathways of recombination by the Mec1 and Mec3 DNA damage sensors and RPA. Nucleic Acids Res. 35 (2007) 822-838.

[34] N. Grandin, C. Damon, M. Charbonneau, Cdc13 prevents telomere uncapping and Rad50-dependent homologous recombination. EMBO J. 20 (2001) 6127-6139.

[35] K. Umezu, N. Sugawara, C. Chen, J.E. Haber, R.D. Kolodner, Genetic analysis of yeast RPA1 reveals its multiple functions in DNA metabolism. Genetics 148 (1998) 989-1005.

[36] V. Lundblad, E.H. Blackburn, An alternative pathway for yeast telomere maintenance rescues est1' senescence. Cell 73 (1993) 347-360.

[37] T.A. Weinert, G.L. Kiser, L.H. Hartwell, Mitotic checkpoint genes in budding yeast and the dependence of mitosis on DNA replication and repair. Genes Dev. 8 (1994) 652-665.

[38] J.S. Searle, K.L. Schollaert,B.J. Wilkins, Y. Sanchez, The DNA damage checkpoint and PKA pathways converge on APC substrates and Cdc20 to regulate mitotic progression. Nat. Cell Biol. 6 (2004) 138-145.

[39] L.H. Hartwell, T.A. Weinert, Checkpoints: controls that ensure the order of cell cycle events. Science 246 (1989) 629-634.

[40] T.A. Weinert, L.H. Hartwell, Cell cycle arrest of cdc mutants and specificity of the RAD9 checkpoint. Genetics 134 (1993) 63-80.

[41] D. Lydall, T. Weinert, Use of cdc13-1-induced DNA damage to study effects of checkpoint genes on DNA damage processing. Methods Enzymol. 283 (1997) 410-424.

[42] M.J. Carson, L. Hartwell, $C D C 17$ : an essential gene that prevents telomere elongation in yeast. Cell 42 (1985) 249-257.

[43] P. Plevani, M. Foiani, M. Muzi Falconi, A. Pizzagalli, C. Santocanale, S. Francesconi, P. Valsasnini, A. Comedini, S. Piatti, G. Lucchini, The yeast DNA polymerase-primase complex: genes and proteins. Biochim. Biophys. Acta 951 (1988) 268-273.

[44] L. Maringele, D. Lydall, EXO1-dependent single-stranded DNA at telomeres activates subsets of DNA damage and spindle checkpoint pathways in budding yeast yku70 $\Delta$ mutants. Genes Dev. 16 (2002)1919-1933.

[45] M.K. Zubko, S. Guillard, D. Lydall, Exo1 and Rad24 differentially regulate generation of ssDNA at telomeres of Saccharomyces cerevisiae cdc13-1 mutants. Genetics 168 (2004) 103-115.

[46] J.M. Dewar, D. Lydall, Pif1- and Exo1-dependent nucleases coordinate checkpoint activation following telomere uncapping. EMBO J. 29 (2010) 4020-4034.

[47] M. Downey, R. Houlsworth, L. Maringele, A. Rollie, M. Brehme, S. Galicia, S. Guillard,M. Partington, M.K. Zubko,N.J. Krogan, A. Emili, J.F. Greenblatt, L. Harrington, D. Lydall, D. Durocher, A genome-wide screen identifies the evolutionarily conserved KEOPS complex as a telomere regulator. Cell 12 (2006)1155-1168. 
[48] S. Le, J.K. Moore, J.E. Haber, C.W. Greider, RAD50 and RAD51 define two pathways that collaborate to maintain telomeres in the absence of telomerase. Genetics 15, (1999)143152.

[49] S.C. Teng, V.A. Zakian, Telomere-telomere recombination is an efficient bypass pathway for telomere maintenance in Saccharomyces cerevisiae. Mol. Cell. Biol. 19 (1999) 8083-8093.

[50] Q. Chen, A. lijpma, C.W. Greider, Two survivor pathways that allow growth in the absence of telomerase are generated by distinct telomere recombination events. Mol. Cell. Biol. 21 (2001) 1819-1827.

[51] H. Takata, Y. Kanoh, N. Gunge, K. Shirahige, A. Matsuura, Reciprocal association of the budding yeast ATM-related proteins Tel1 and Mec1 with telomeres in vivo. Mol. Cell 14 (2004) 515-522.

[52] D. Bosoy, Y. Peng, I. Saira Mian, N.F. Lue, Conserved N-terminal motifs of telomerase reverse transcriptase required for ribonucleoprotein assembly in vivo. J. Biol. Chem. 278 (2003) 3882-3890.

[53] N. Grandin, M. Charbonneau, The Rad51 pathway of telomerase-independent maintenance of telomeres can amplify $\mathrm{TG}_{1-3}$ sequences in yku and cdc13 mutants of Saccharomyces cerevisiae. Mol. Cell. Biol. 23 (2003) 3721-3734.

[54] V. Lundblad, J.W. Szostak, A mutant with a defect in telomere elongation leads to senescence in yeast. Cell 57 (1989) 633-643.

[55] J. Smith, H. Zou, R. Rothstein, Characterization of genetic interactions with RFA1: the role of RPA in DNA replication and telomere maintenance. Biochimie 82 (2000) 71-78.

[56] V. Schramke, P. Luciano, V. Brevet, S. Guillot, Y. Corda, M.P. Longhese, E. Gilson, V. Géli, RPA regulates telomerase action by providing Est1p access to chromosome ends. Nat. Genet. 36 (2004) 46-54.

[57] P. Luciano, S. Coulon, V. Faure, Y. Corda, J. Bos, S.J. Brill, E. Gilson, M.N. Simon, V. Géli, RPA facilitates telomerase activity at chromosome ends in budding and fission yeasts. EMBO J. 31 (2012) 2034-2046.

[58] M.J. McEachern, J.E. Haber, Break-induced replication and recombinational telomere elongation in yeast. Annu. Rev. Biochem. 75 (2006) 111-135.

[59] J. Hu, S.S. Hwang, M. Liesa, B. Gan, E. Sahin, M. Jaskelioff, Z. Ding, H. Ying,A.T. Boutin,H. Zhang, S. Johnson, E. Ivanova, M. Kost-Alimova, A. Protopopov,Y.A. Wang, O.S. Shirihai, L. Chin, R.A. DePinho, Antitelomerase therapy provokes ALT and mitochondrial adaptive mechanisms in cancer. Cell 148 (2012) 651-663.

[60] J.B. Vannier, V. Pavicic-Kaltenbrunner, M.I.R. Petalcorin, H. Ding, S.J. Boulton, RTEL1 dismantles $\mathrm{T}$ loops and counteracts telomeric G4-DNA to maintain telomere integrity. Cell 149 (2012) 795-806. 
[61] Y. Wang, C.D. Putnam, M.F. Kane, W. Zhang, L. Edelmann, R. Russell, D.V. Carrión, L. Chin, R. Kucherlapati, R.D. Kolodner, W. Edelmann, Mutation in Rpa1 results in defective DNA double-strand break repair, chromosomal instability and cancer in mice. Nat. Genet. 37 (2005) 750-755.

[62) C. Chen, K. Umezu, R.D. Kolodner, Chromosomal rearrangements occur in $S$. cerevisiae rfa1 mutator mutants due to mutagenic lesions processed by double-strand-break repair. Mol. Cell 2 (1998) 9-22.

[63] J. Graumann, L.A. Dunipace, J.H. Seol, W.H. McDonald, J.R.3 ${ }^{\text {rd }}$ Yates, B.J. Wold, R.J. Deshaies, Applicability of tandem affinity purification MudPIT to pathway proteomics in yeast. Mol. Cell. Proteomics 3 (2004) 226-237.

[64] K. Myung, C. Chen, R.D. Kolodner, Multiple pathways cooperate in the suppression of genome instability in Saccharomyces cerevisiae. Nature 411 (2001) 1073-1076.

[65] A. Bochkarev, R.A. Pfuetzner, A.M. Edwards, L. Frappier, Structure of the singlestranded-DNA-binding domain of replication protein A bound to DNA. Nature 385 (1997) 176181.

[66] M.D. Rose, P. Novick, J.H. Thomas, D. Botstein, G.R. Fink, A Saccharomyces cerevisiae genomic plasmid bank based on a centromere-containing shuttle vector. Gene 60 (1987) 237-243.

[67] R.D. Gietz, A. Sugino, New yeast-Escherichia coli shuttle vectors constructed with in vitro mutagenized yeast genes lacking six-base pair restriction sites. Gene 47 (1988) 527534.

[68] Z. Sun, D.S. Fay, F. Marini,M. Foiani, D.F. Stern, Spk1/Rad53 is regulated by Mec1dependent protein phosphorylation in DNA replication and damage checkpoint pathways. Genes Dev. 10 (1996) 395-406. 


\section{Legends of figures}

Figure 1. Mutants of RFA1 deregulated in telomere uncapping-induced cell cycle arrest. (A, B) Telomeric recombination, induced here by inactivating TLC1, coding for the RNA subunit of telomerase, partially rescued the temperature sensitivity of the cdc13-1 mutation $(\mathbf{A})$, an event accompanied by telomeric recombination assessed by Southern blot (B). The smear at 1.3-1.4 $\mathrm{Kb}$ represents the average length of the telomeres. The pattern of recombination is typical of type II recombination, amplifying the $\mathrm{TG}_{1-3}$ telomeric repeats [49]. Note that in the cdc13-1 background, type II is the only pathway permitted [53]. (C) Deregulation of cell cycle control upon cdc13-1-induced telomere uncapping was compared in checkpoint-deficient mutants, rad24 deletion and rfa1-t11, and other rfa1 mutants. All rfa1 mutants were under the control of natural promoter in a low-copy plasmid (centromeric, of the YCplac series, expressed at one or two copies per cell; ref. 66,67) in an rfa1 deletion background (RFA1 is an essential gene). Last row corresponds to the rfa1 deletion strain transformed with wildtype RFA1 as a control. Shown schematically is the fate of cdc13-1 mutant cells with or without an intact checkpoint at the indicated temperatures.

Figure 2. The DNA damage checkpoint is intact in telomere-uncapped cdc13-1 rfa1 mutant cells. (A) Activation of the checkpoint kinase Rad53 upon cdc13-1-induced telomere uncapping $\left(2 \mathrm{~h}, 34^{\circ} \mathrm{C}\right)$ was assessed by visualizing electrophoretic mobility shifts of the protein [68]. Strains expressed $R A D 53-\mathrm{HA}_{2}$ from the $R A D 53$ genomic locus under the control of native promoter and the protein was detected following immunoprecipitation and Western blotting using monoclonal anti-HA antibody. The cdc13-1 mec1 sm/1 deletion, cdc13-1 mec32 and cdc13-1 rad9 deletion mutants served as controls, Mec1, Mec3 and Rad9 being essential for Rad53 activation. All cdc13-1 rfa1 mutants behaved like checkpoint-proficient cdc13-1 cells and cdc13-1 cells harboring wild-type RFA1, thus indicating an absence of Rad53-mediated checkpoint defect in these mutants. cdc13-1 rfa1-t11 mutant cells were partially defective in activating Rad53, as described previously [25]. (B) In cell viability assays, deregulation in damage-induced cell cycle control provoked by the rfa1 mutations (improved growth at $29^{\circ} \mathrm{C}$ compared with cdc13-1 alone) was found to be additive with the deregulation provoked by checkpoint inactivation in the mec3 deletion background, as combination of mec3 and rfa1 mutations (cdc13-1 mec3 rfa1 triple mutants) rendered this deregulation more severe than in either class of the double mutants, an effect best seen at $30^{\circ} \mathrm{C}$. (C) At the restrictive temperature for growth for cdc $13-1$ of $34^{\circ} \mathrm{C}$, the arrest of checkpoint-deficient cells, here cdc13-1 rad24 $\Delta$, differed from that in cdc13-1 rfa1-9, thus suggesting that the reasons for improved growth at $29^{\circ} \mathrm{C}$ (see Figure 1) were not the same for the two strains. cdc13-1 rad24 $\Delta$ cells exhibited a typical override of the DNA damageinduced block to cell cycle progression, indicated by re-budding, while the cdc13-1 rfa1-9 
cells continued to enlarge in size without re-budding. (D) The growth defects of the temperature-sensitive cdc17-1 mutant (Cdc17 is a subunit of DNA polymerase alphaprimase) were exacerbated by the checkpoint-deficient $d d c 1 \Delta$ and $r f a 1-t 11$ mutations, but not by the rfa1-12 mutation, thus suggesting that the later mutation unlike the former two ones does not confer defect in sensing the damage at the replication forks provoked by cdc17-1.

Figure 3. The rfa1 mutations do not rescue cdc13-1 by diminishing Exo1-induced telomeric DNA degradation from double- to single-stranded. (A) Cell viability assays (spot tests) of cdc13-1 mutant strains of the indicated relevant genotypes at the indicated temperatures. The triple cdc13-1 rfa1 exo1s mutant grew better than the double cdc13-1 rfa1 and the cdc13-1 exo1 $\Delta$ double mutants, an effect best visible at $30^{\circ} \mathrm{C}$, suggesting that the rescue of cdc13-1 by the rfa1 mutations did not depend on Exo1. (B) Detection of abnormally high levels of telomeric ssDNA in the cdc13-1 strains of the indicated relevant genotypes, following growth at $34^{\circ} \mathrm{C}$ for $2 \mathrm{~h}$, was performed by Southern blot analysis under native conditions (right panel). Only strains harboring the exo1s mutation exhibited a clear diminution of telomeric damage, thus indicating that the rfa1 mutations do not rescue cdc131 through a failure to activate Exo1.

Figure 4. The rfa1-12 mutation triggers telomeric recombination in cdc13-1 cells with uncapped telomeres. (A) Cells expressing the indicated rfa1 mutations either in an otherwise wild-type background (left) or in the cdc13-1 background (right) were grown at $24^{\circ} \mathrm{C}$ and telomere organization assessed by Southern blotting with a telomeric probe. A wild-type $R F A 1^{+}$strain is illustrated as a control (wt). Following digestion of genomic DNAs with Xhol, the smear migrating at 1.1-1.4 $\mathrm{Kb}$ represents the average length of most telomeres, those containing $Y^{\prime}$ subtelomeric regions. From non $Y^{\prime}$-chromosomes, Xhol cutting typically generates fragments migrating at $\sim 2.1,2.3,3.2$ and $3.9 \mathrm{~Kb}$ in Southerns. (B) The cdc13-1 rfa1-12 mutant exhibited telomere recombination following growth at $29^{\circ} \mathrm{C}$ on solid medium, here after 5 passages, one passage corresponding to restreaking of single colonies every 3 days ( $\sim 30$ cell divisions per passage at $29^{\circ} \mathrm{C}$ ). Meanwhile, increasing telomere uncapping by growing the other cdc13-1 rfa1 strains at $29^{\circ} \mathrm{C}$ (compared with growth at $24^{\circ} \mathrm{C}$ in panel A) led to substantial changes in telomere length and organization, different from one rfa1 mutant to the next, but, unlike with the cdc13-1 rfa1-12 mutant, not to telomeric recombination.

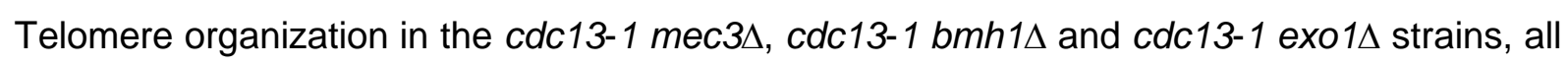
exhibiting deregulated growth at $29^{\circ} \mathrm{C}$ (see main text), is shown for comparison. 
Figure 5. Improved growth in cdc13-1 rfa1-12 can take place in the absence of Rad52dependent telomeric recombination. (A) cdc13-1 rfa1-12 cells exhibited deregulated growth at $29^{\circ} \mathrm{C}$ even the absence of telomere recombination due to $R A D 52$ deletion. (B) Kinetics for reaching telomere recombination upon telomere uncapping, at $27.5^{\circ} \mathrm{C}$, in the $c d c 13-1 \mathrm{rfa} 1-12$ mutant cells. Sister cells deleted for RAD52 were grown and analyzed in parallel. See main text for more detail on the experimental set-up.

Figure 6. Tel1 kinase activity and EST2/telomerase are required for the deregulated growth conferred by the rfa1 mutations upon telomere uncapping by cdc13-1. (A) Deletion of TEL1 suppressed improved growth of the cdc13-1 rfa1-12 mutant and partially suppressed that of cdc13-1 rfa1-1. (B) Top panels: Expression of a kinase dead allele of tel1 (tel1-kd) from TEL1 genomic locus also suppressed improved growth of the cdc13-1 rfa1 mutants to various extents depending on the rfa1 mutation. Bottom panel: In the absence of incurred damage, the simultaneous presence of one of the analyzed rfa1 mutations and of the tel1-kd mutation did not interfere with cell growth. (C) Telomere length was measured by Southern blot $\left(\mathrm{P}^{32}-\mathrm{TG}_{1-3}\right.$ probe, genomic DNA digested with Xhol) in strains with the indicated relevant genotypes. The est2-DF338 mutation conferred roughly equal telomere shortening in all rfa1 mutant strains, as well as in the wild type (left panel). Right panel shows telomere shortening in tel1 and rad50 null strains for comparison. (D) The est2-DF338 mutation suppressed deregulated improved growth conferred by the rfa1 mutations in cdc13-1 mutants with uncapped telomeres, at 27.5 or $29^{\circ} \mathrm{C}$.

Figure 7. Growth advantage conferred by the rfa1-1, rfa1-9, rfa1-10, rfa1-1 and rfa1-12 mutations is not additive with that conferred by telomeric recombination. Telomeric recombination (as shown in Figure 1B) in post-senescence type II survivors, generated following genetic inactivation of telomerase (t/c1 disruption alone or in conjunction with the est2-DF338 mutation), led to improved growth at $27.5-29^{\circ} \mathrm{C}$ as expected (see Figure $1 \mathrm{~A}, \mathrm{~B}$ ). However, under these conditions the rfa1-1 mutants no longer grew better than the sister $R F A 1^{+}$cells. 
Table 1. Sequence analysis of the amino acid changes in the rfa1 alleles analyzed in the present study*.

Rfa1-1: I89T, T116S, S160P, D240G, T346P, D415N, D465V, T510S, E562G, Y575C

Rfa1-9: S108P, H299D, N310S, V317I, N368Y, S487T, Q571R

Rfa1-10: F269L

Rfa1-11: F238L, Q428R

Rfa1-12: L227S

* Rfa1-1 and Rfa1-9 mutations have already been reported in Grandin and Charbonneau (2007), but are also shown here for convenience. 

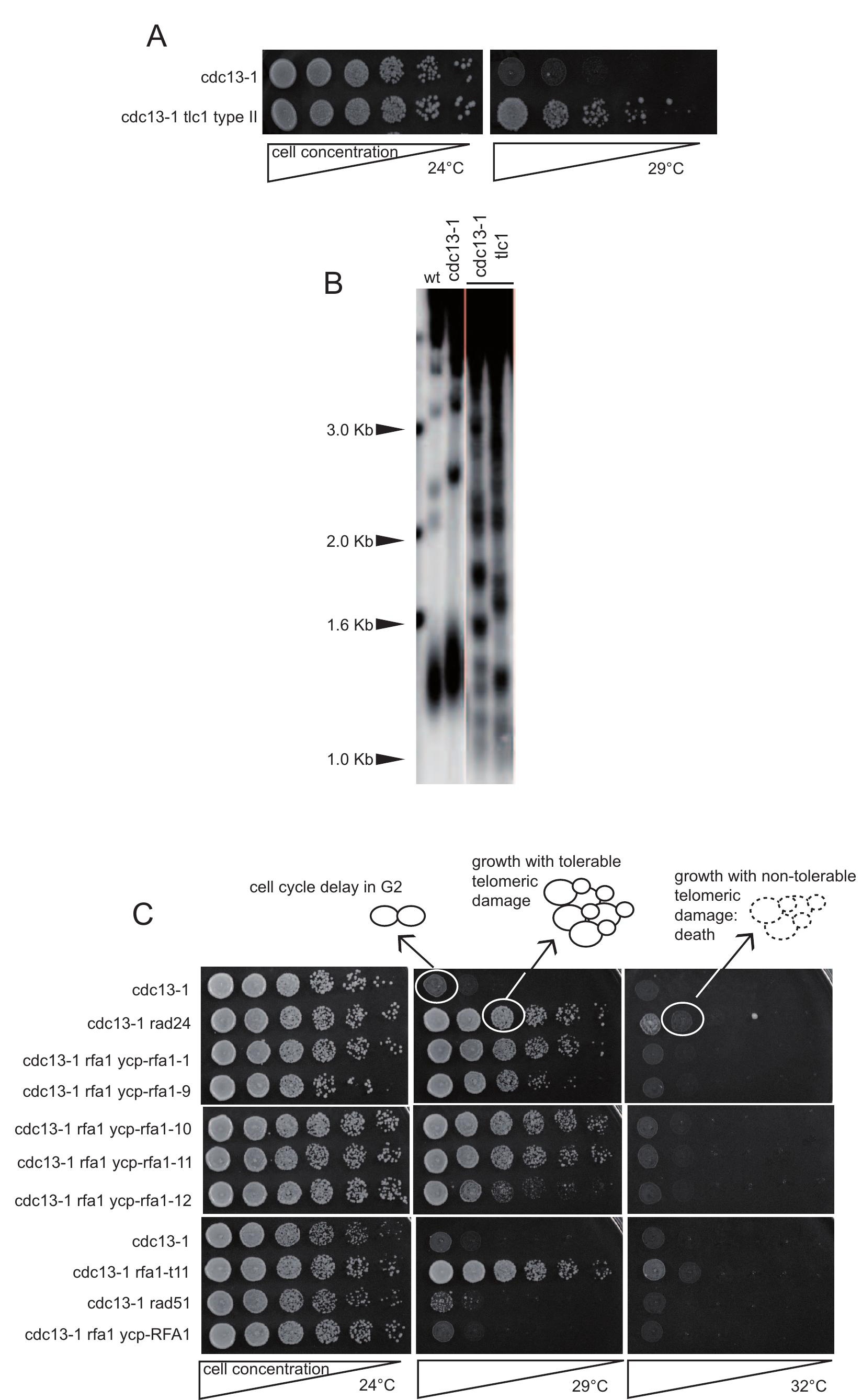

Figurare 1

.

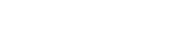

.

.

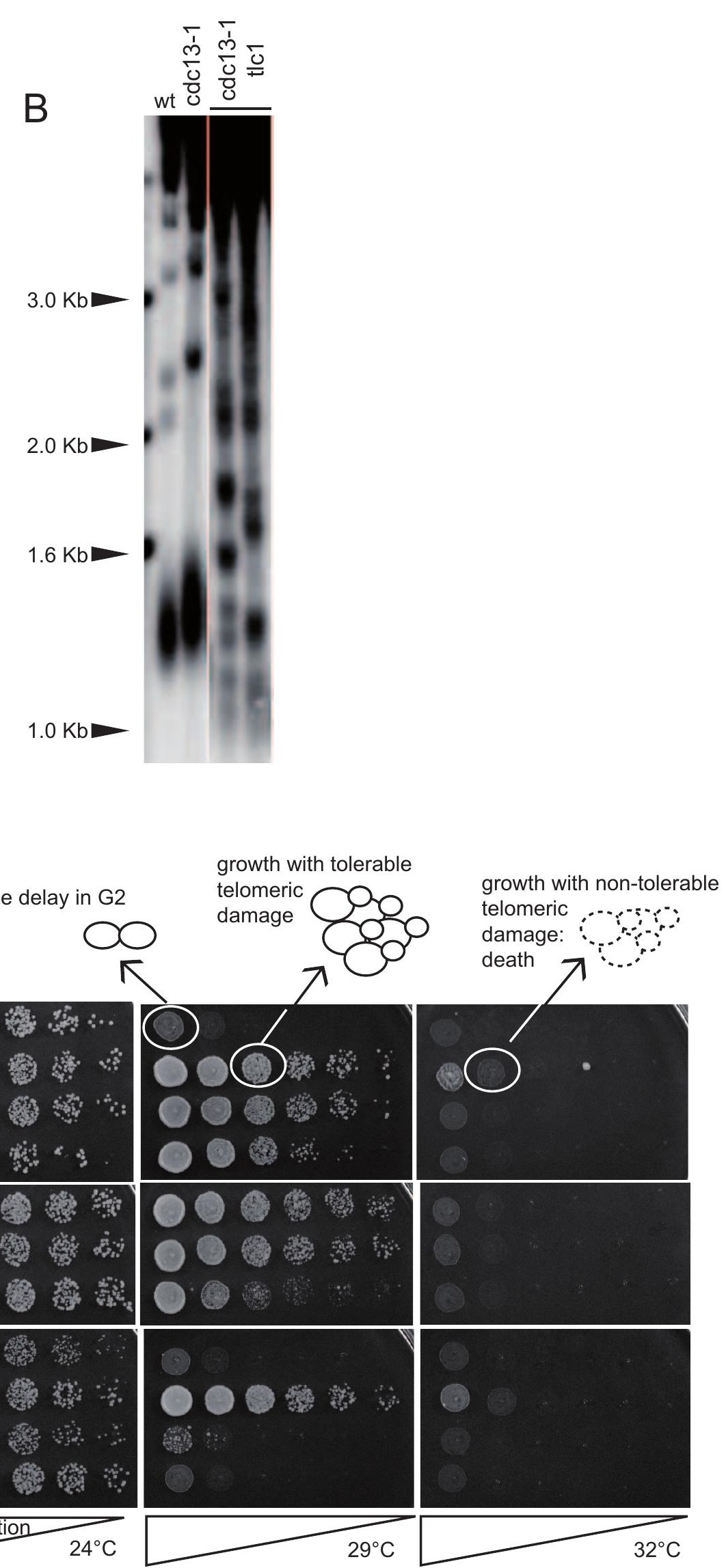

$$
\infty
$$
dc13-1 rfa1 ycp-rfa1-11$$
\text { cdc13- }
$$
cdc13-1 rad51

\section{$\mathrm{cd}$}


A

RAD53-HA

RAD53-HA

cdc13-1 rfa1 уср-

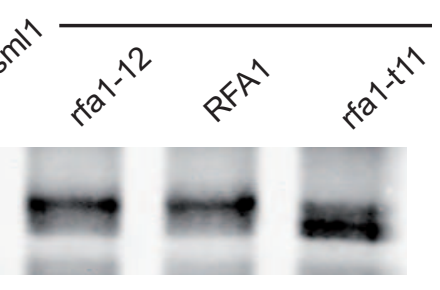

cdc13-1 rfa1 ycp-

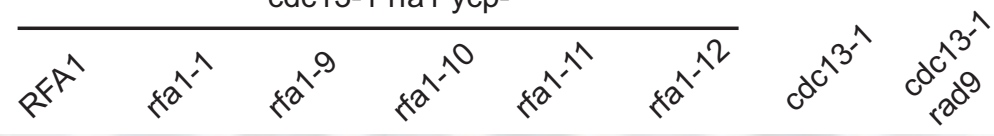

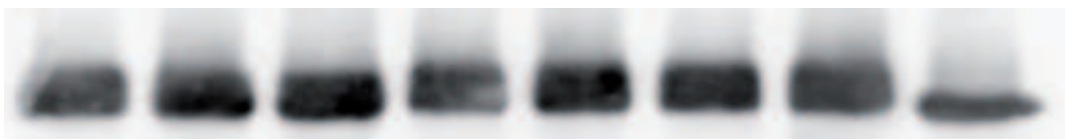

B

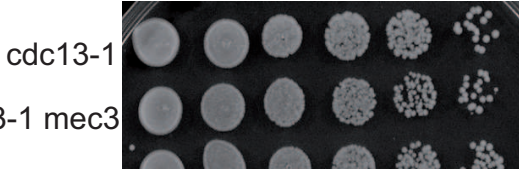
rfa1 ycp-rfa1-1 $\bigcirc \bigcirc \bigcirc$. क्षे cdc13-1 rfa1 ycp-rfa1-1 cdc13-1 mec3 rfa1 ycp-rfa1-1 cdc13-1 rfa1 ycp-rfa1-9 cdc13-1 mec3 rfa1 ycp-rfa1-9 cdc13-1 cdc13-1 mec3 cdc13-1 rfa1 ycp-rfa1-10 cdc13-1 mec3 rfa1 ycp-rfa1-10 cdc13-1 rfa1 ycp-rfa1-12 cdc13-1 mec3 rfa1 ycp-rfa1-12 0020 की
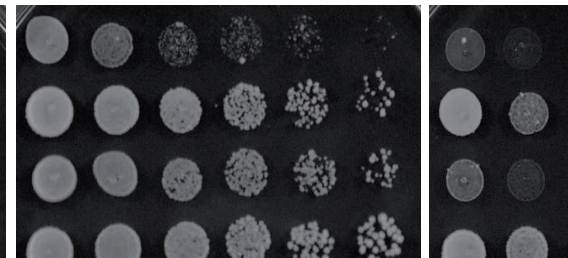
000 순 00003 0000 . 10000 \% 10000 ㄴ. 20000 海 2000 䊉

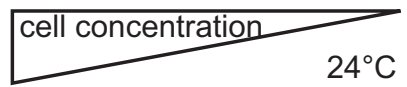

$24^{\circ} \mathrm{C}$
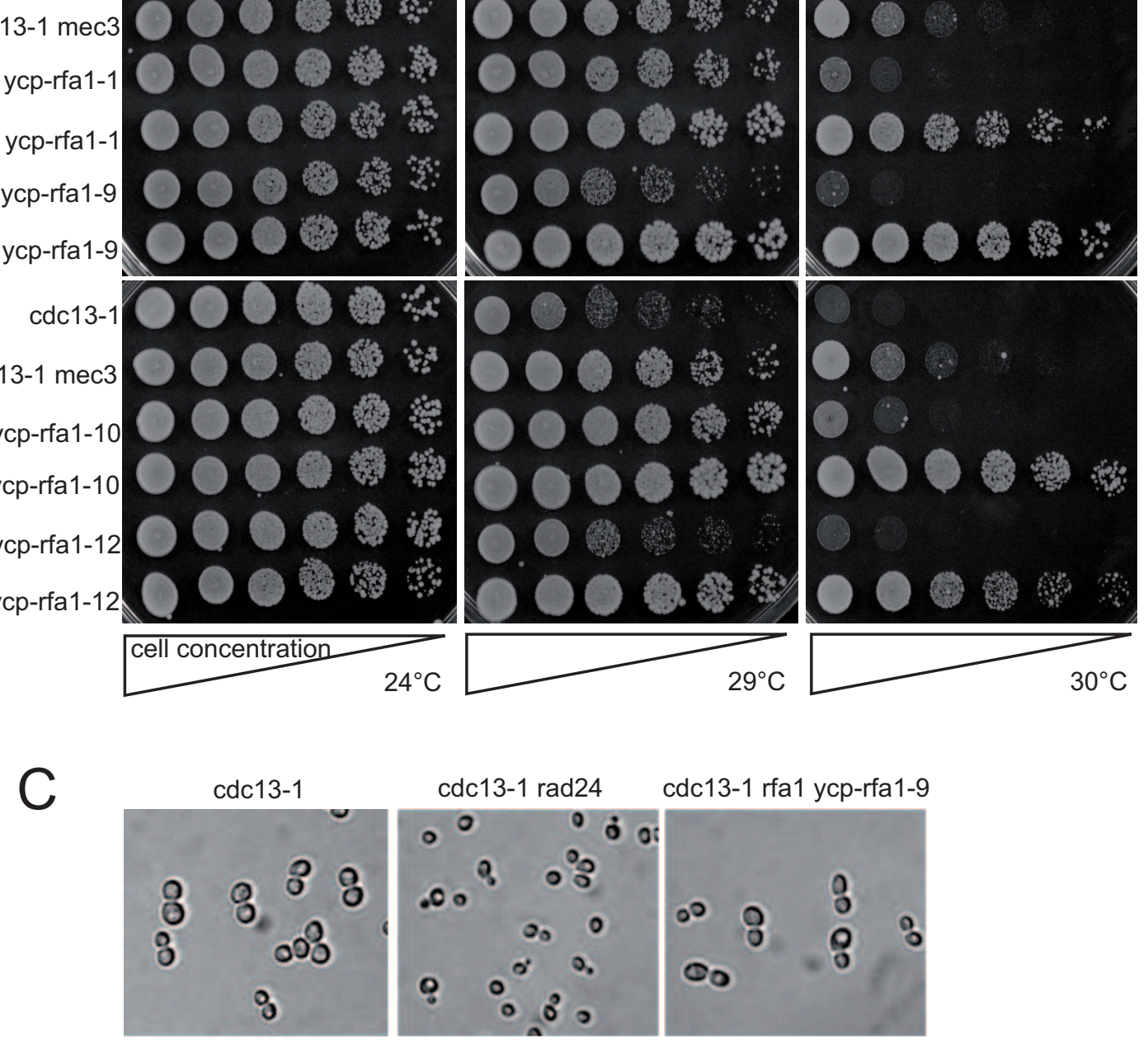

$2 \mathrm{hr}, 34^{\circ} \mathrm{C}$

\section{D}
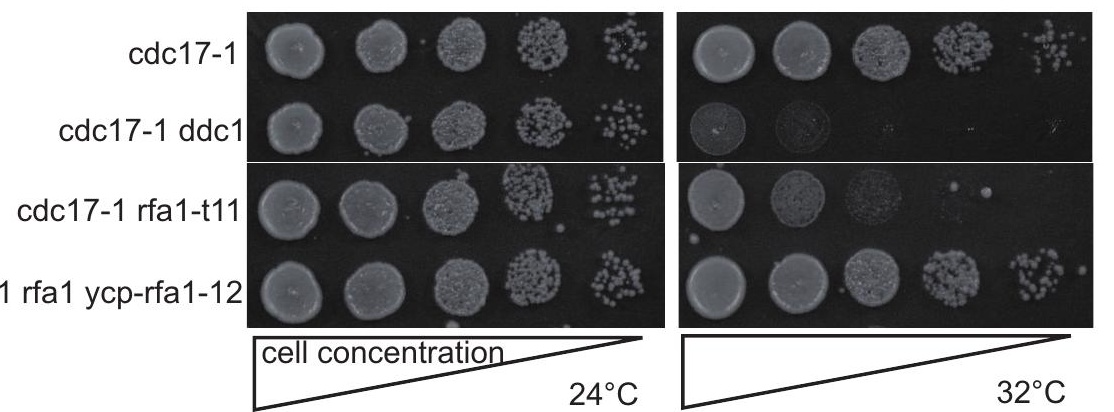
Figguße 3

A

cdc13-1

cdc13-1 rfa1 ycp-rfa1-1

cdc13-1 rfa1 ycp-rfa1-9

cdc13-1 rfa1 ycp-rfa1-10

cdc13-1 rfa1-12

cdc13-1 exo1

dc13-1 exo1 rfa1 ycp-rfa1-1

cdc13-1 exo1 rfa1 ycp-rfa1-9

cdc13-1 exo1 rfa1 ycp-rfa1-10

cdc13-1 exo1 rfa1-12
$000.0 \%$

00.00 .6

000 व

0000.0

0008 綡

10000 然

0009

000 .

0009 a

$0000.4 \%$

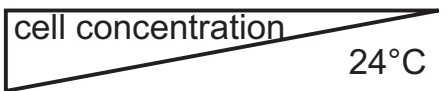

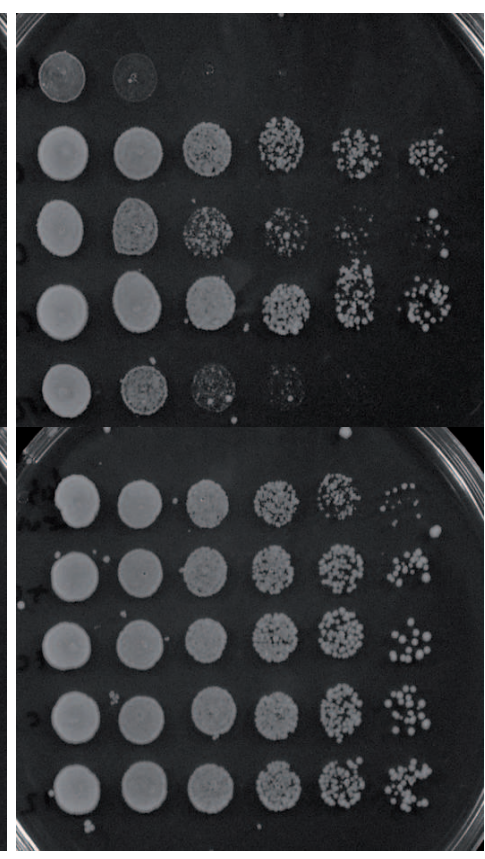

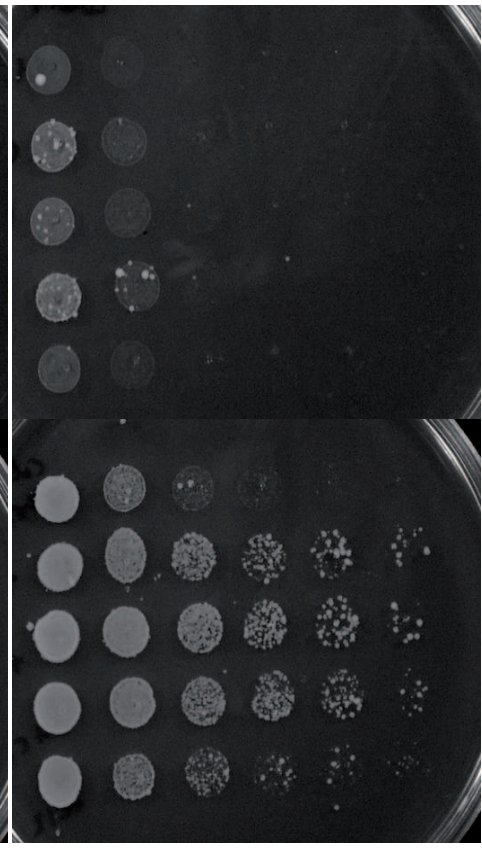

$29^{\circ} \mathrm{C}$

$30^{\circ} \mathrm{C}$

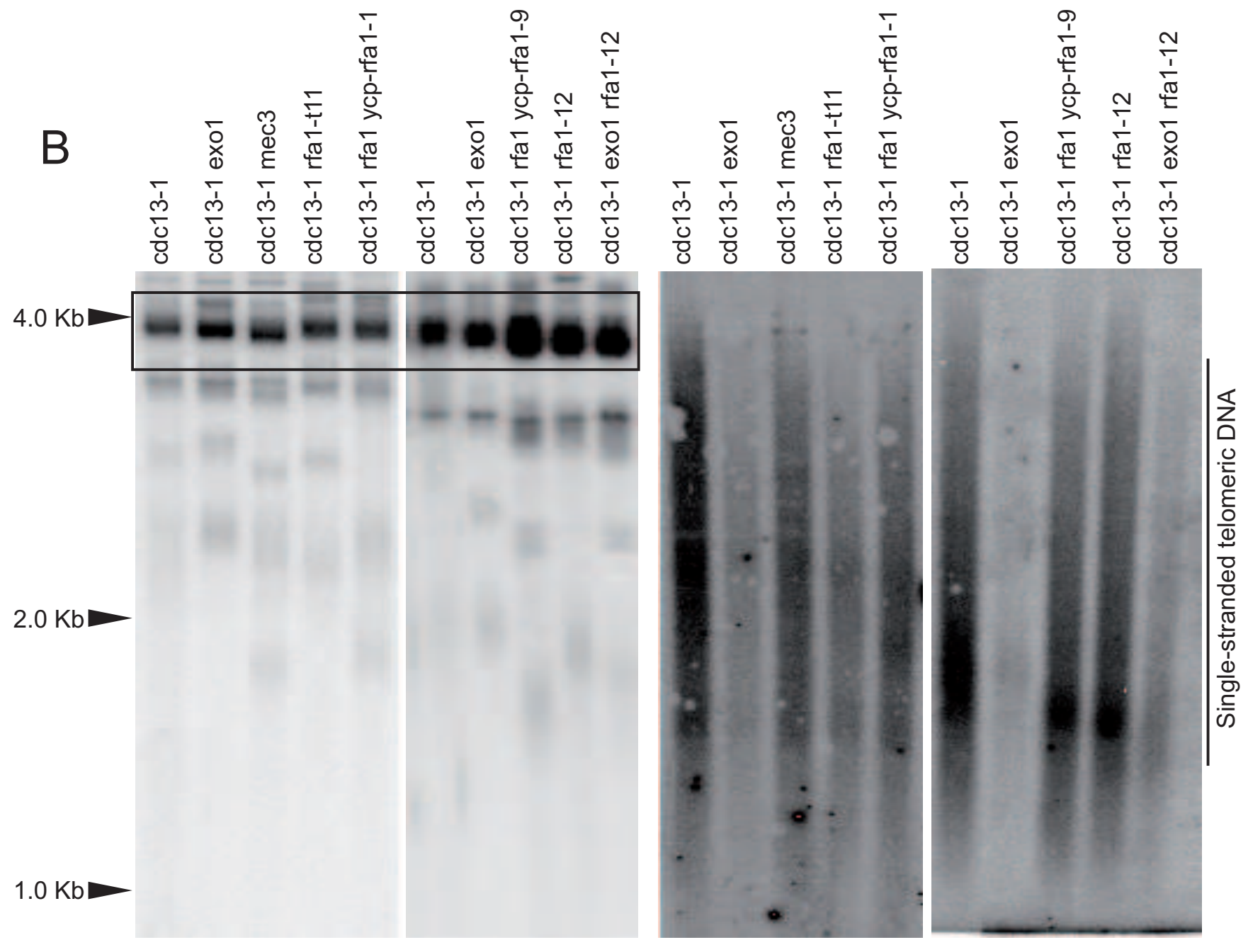



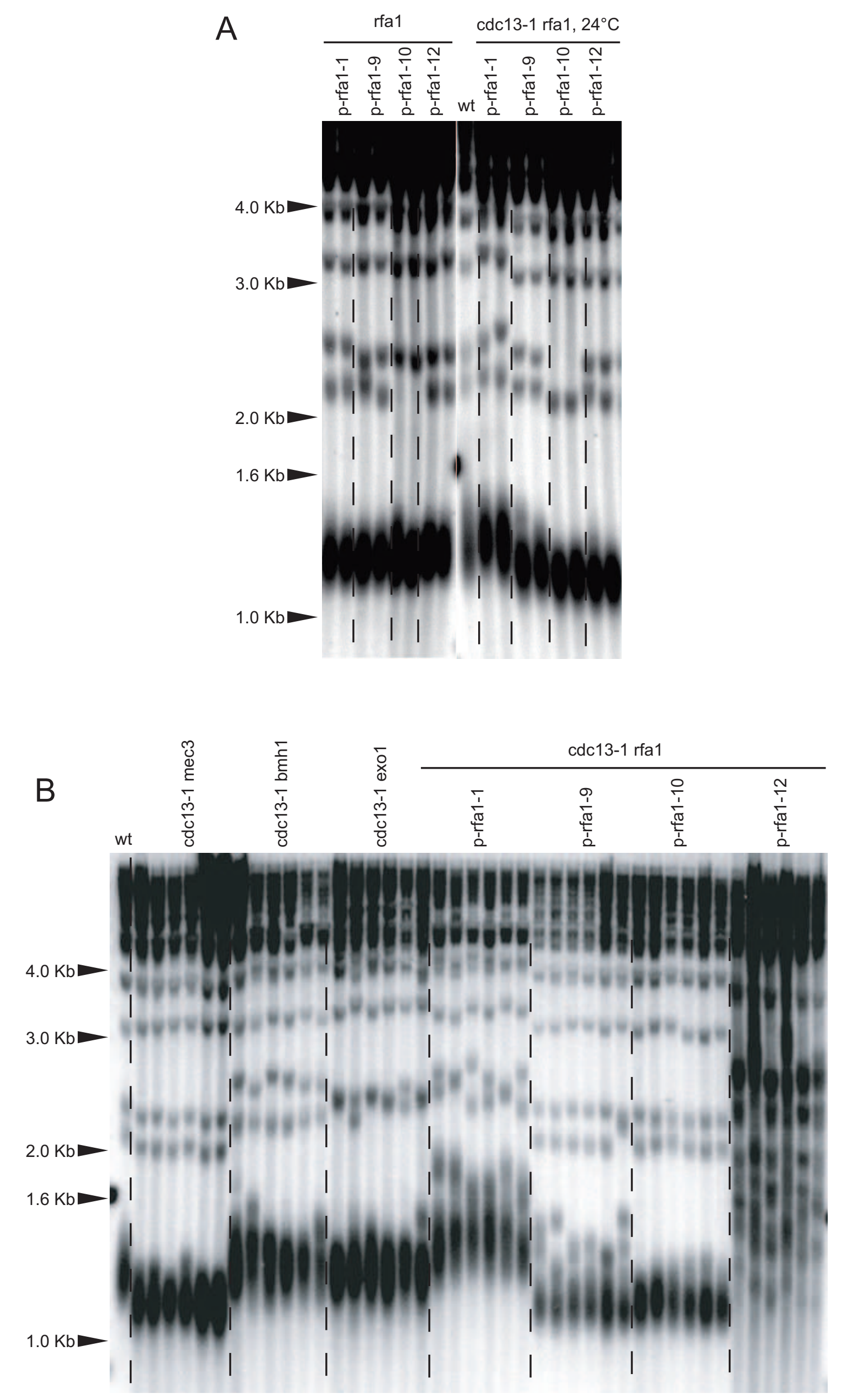
3 days of growth after selection of the spores bearing the relevant genotype

A
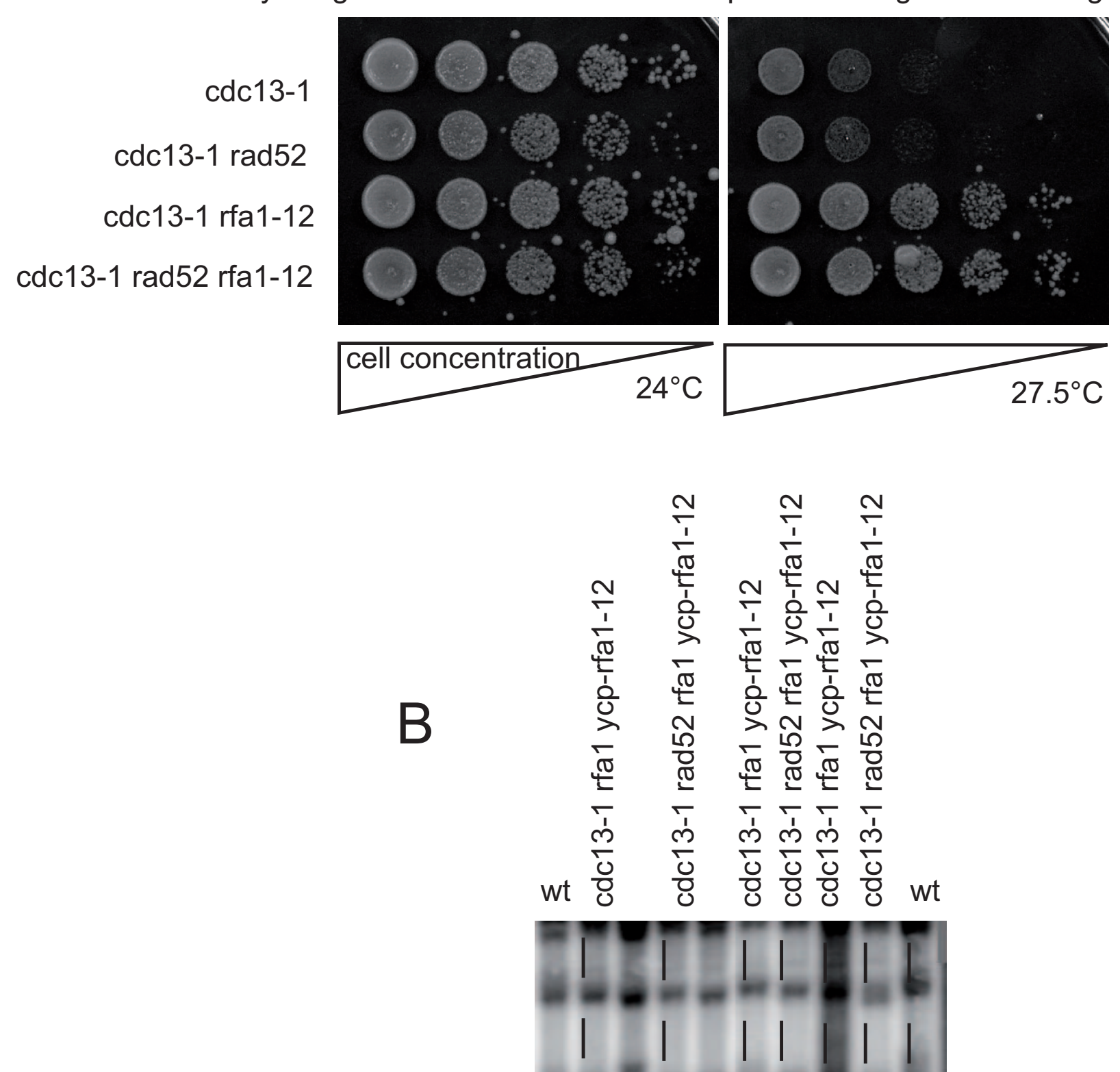

$3.0 \mathrm{~Kb}-\mathrm{|} \quad \mathrm{|}|\mathrm{|}|$

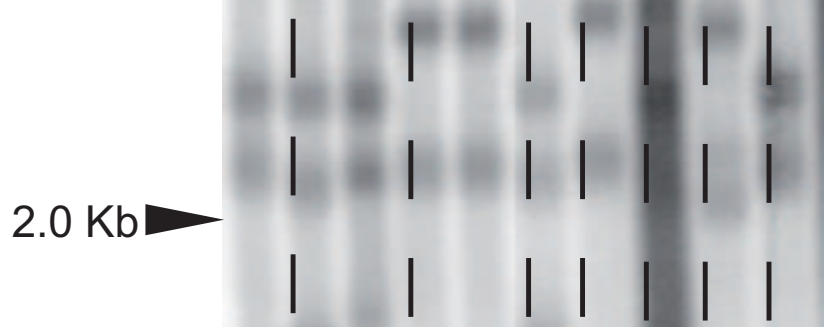

$1.6 \mathrm{~Kb} \quad|\quad| 1 \mid$

Number of days of growth after selection of the spores bearing the relevant genotype:
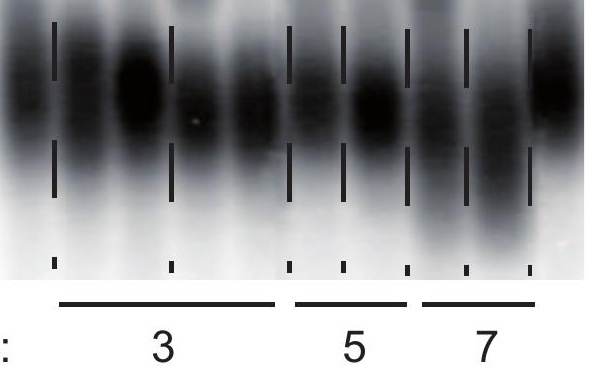
Figurat,6

dc13-1 tel1

cdc13-1 rfa1 ycp-rfa1-1 cdc13-1 tel1 rfa1 ycp-rfa1-1

cdc13-1 rfa1 ycp-rfa1-12

cdc13-1 tel1 rfa1 ycp-rfa1-12

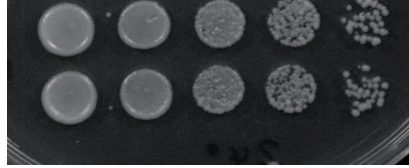

cell concentration

$24^{\circ} \mathrm{C}$

(1)
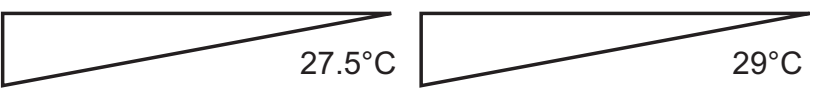

\section{B}

\section{cdc13-1}

cdc13-1 tel1-kd

cdc13-1 rfa1 ycp-RFA1

cdc13-1 tel1-kd rfa1 ycp-RFA1

cdc13-1 rfa1 ycp-rfa1-9

cdc13-1 tel1-kd rfa1 ycp-rfa1-9

cdc13-1 rfa1 ycp-rfa1-10

cdc13-1 tel1-kd rfa1 ycp-rfa1-10

cdc13-1 rfa1-12

cdc13-1 tel1-kd rfa1-12

cdc13-1 rfa1 ycp-rfa1-1

cdc13-1 tel1-kd rfa1 ycp-rfa1-1

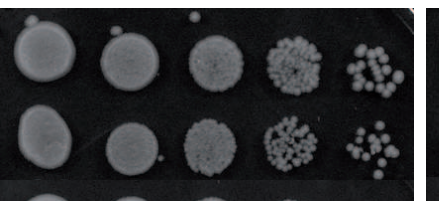

\section{(1)}
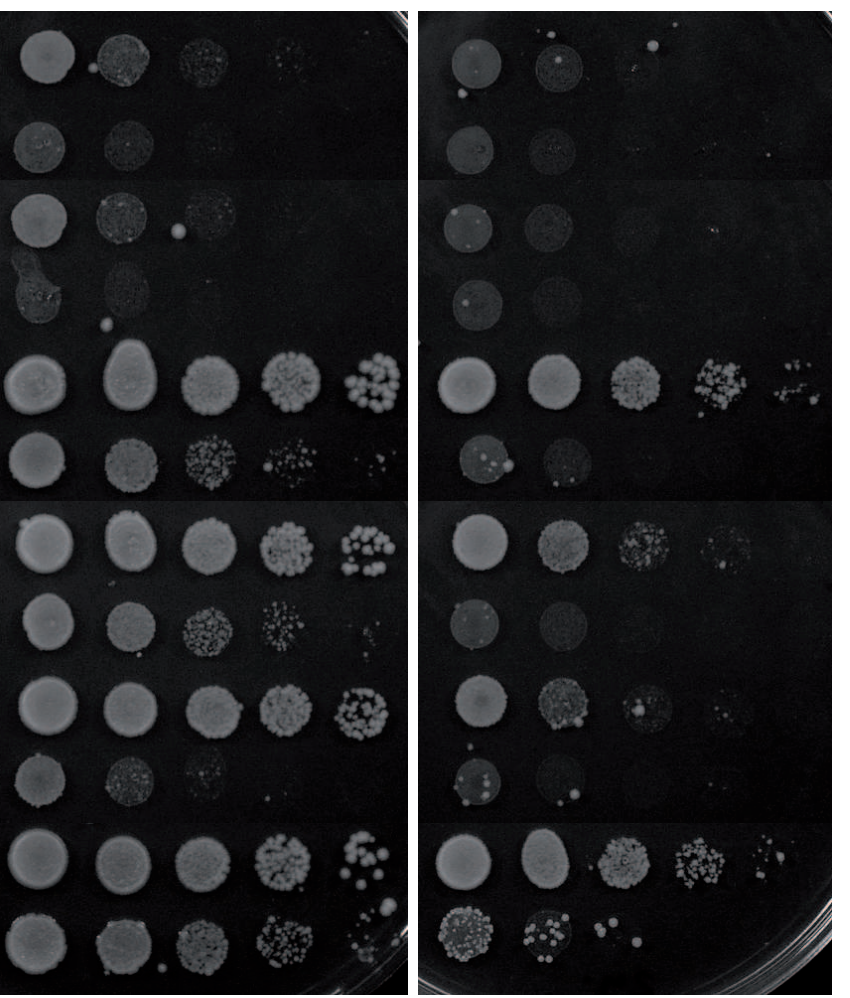

cell concentration $24^{\circ} \mathrm{C}$
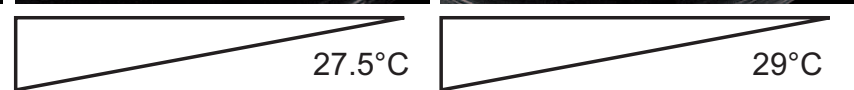

rfa1 ycp-RFA1

tel1-kd rfa1 ycp-RFA1

rfa1 ycp-rfa1-9

tel1-kd rfa1 ycp-rfa1-9

rfa1 ycp-rfa1-10

tel1-kd rfa1 ycp-rfa1-10

rfa1-12

tel1-kd rfa1-12

rfa1 ycp-rfa1-1

tel1-kd rfa1 ycp-rfa1-1

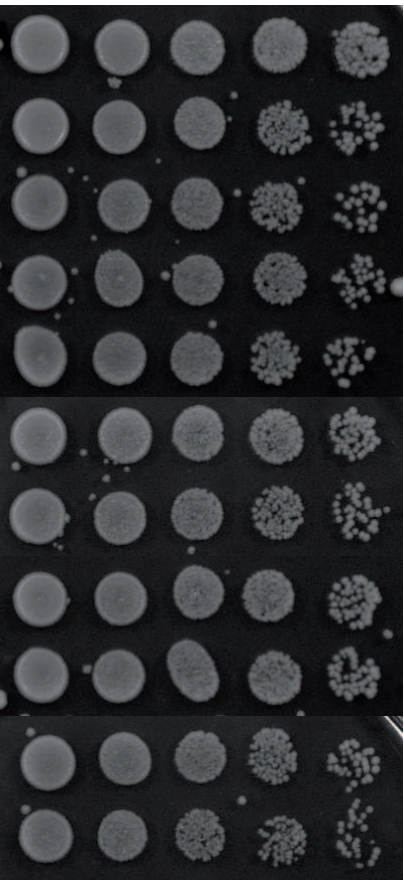

cell concentration 

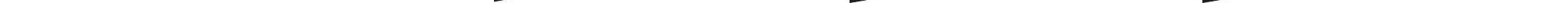


\section{Figure 7}

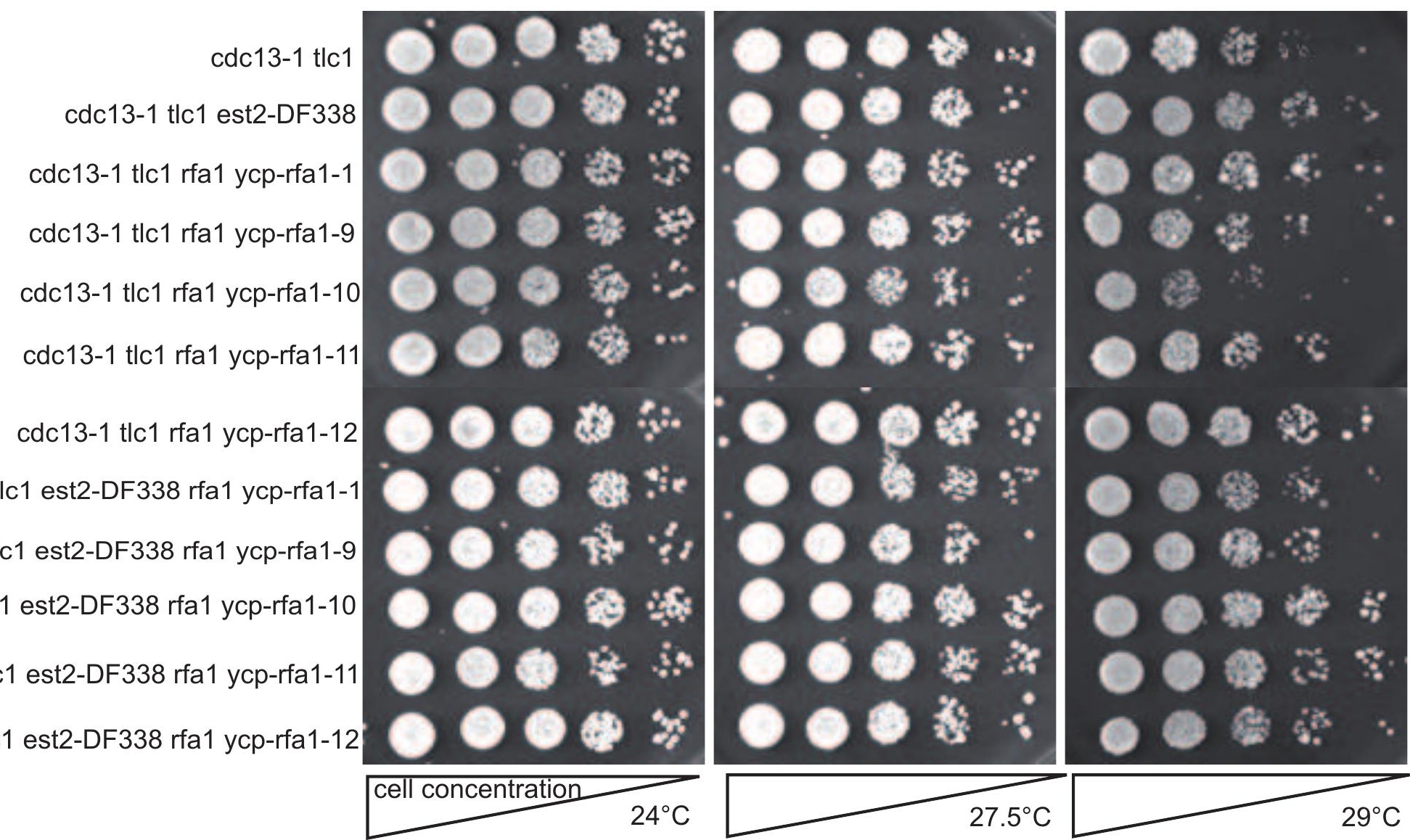

cdc13-1 tlc1 est2-DF338 rfa1 ycp-rfa1-1 cdc13-1 tlc1 est2-DF338 rfa1 ycp-rfa1-9 cdc13-1 tlc1 est2-DF338 rfa1 ycp-rfa1-10 cdc13-1 tlc1 est2-DF338 rfa1 ycp-rfa1-11 cdc13-1 tlc1 est2-DF338 rfa1 ycp-rfa1-12 